DGfE Kongress in Zürich, März 2004

AG Medien und Migration

\title{
Medienpädagogische Praxisforschung mit Kindern und Jugendlichen aus Migrationskontexten
}

\author{
HORST NIESYTO \& PETER HOLZWARTH
}

\section{Ausgangsüberlegungen}

Bisherige Studien zur Mediennutzung von Migranten betonen oft die Existenz generationenspezifischer Mediennutzungsstile. So lässt sich als Trend im Bereich der Fernsehnutzung formulieren, dass ältere Migrantengenerationen noch mehr die muttersprachlichen Satellitenprogramme schauen als jüngere Migrantengenerationen, die eher als ihre Eltern dazu neigen, Programme aus den jeweiligen Aufenthaltsländern zu nutzen (zusammenfassend: Buckingham 2002, 110 f.). Dieser Trend ist jedoch nicht ungebrochen. Gerade bei Kindern und Jugendlichen aus jüngeren Migrantengenerationen ist ein breiter gestreutes Mediennutzungsprofil zu beobachten, das eher auf einen Mix aus unterschiedlichen Programmangeboten hinweist (Programme aus dem Herkunftsland, aus dem aktuellen Aufenthaltsland, verschiedene internationale Programmangebote). Die Auswahl und die Nutzung der Programme hängt mit vielen Faktoren zusammen, wobei eigene Beobachtungen und Studien besonders die große Bedeutung globalisierter, populärer Musikangebote für Jugendliche verdeutlichen, und zwar sowohl im Bereich der Mediennutzung als auch im Kontext des Selbstausdrucks mit Medien (Niesyto 2003: 61 f.).

Bislang gibt es nur wenige Analysen, die die Mediennutzung von Kindern und Jugendlichen aus Migrationskontexten unter Aspekten der symbolischen Verarbeitung und der Bedeutung der Medienangebote für Identitätsbildungsprozesse untersuchen. Buckingham (ebd.) weist darauf hin, dass in diesem Bereich oft mit suggestiven Hypothesen gearbeitet werde. So würden z.B. Aksoy und Robbins (2000) unterstellen, dass sich Medienrezeptionserfahrungen von Migranten wesentlich von Nicht-Migranten unterscheiden. Ähnlich wie bei Hannerz (1996) und Cunningham (2001) werde argumentiert, dass Kinder und Jugendliche aus Migrationskontexten bei der Mediennutzung "hybride“ und „kosmopolitische“ Identitäten ausbilden. Demgegenüber betont Buckingham:

„Generally speaking, however, we still have very little evidence about how migrant children make sense of the range of media representations available to them. For many, their media experiences are likely to be a complex mixture of the global and the local. They may share aspects of global media culture (Disney or Pokémon) with children from the host culture - even though these texts may be equally alien to both groups. Ironically, such material may provide just as much connection with their memories of their home country as the programming on specialist satellite channels. The particular combination of cultural specificity and universality ('otherness' and 'sameness') of a global production like The Simpsons may paradoxically unite Turkish children living in 
Germany both with their German peers and with their cousins back home in Turkey and potentially with children in England or in Hong Kong or in Nigeria. Quite how they might interpret such a text - not least (in this instance) in the light of their own very different experiences of family life - and how they might discuss it with their friends and family is the kind of issue that researchers have barely begun to address" $(2002,111)$.

In Deutschland haben sich in den späten 80er und in den 90er Jahren Ansätze der Medienrezeptionsforschung herausgebildet, die versuchen, den komplexen Vermittlungsprozessen von Medienangeboten, subjektiver Mediennutzung und soziokulturellen Kontexten auf die Spur zu kommen. Das Thema „Medien und Identität“ wurde dabei insbesondere von der strukturanalytischen Rezeptionsforschung (u.a. Charlton/Neumann 1992) sowie von verschiedenen Studien im Bereich der „Cultural Studies“ (vgl. diverse Beiträge in dem Sammelband von Winter/Thomas/Hepp 2003) untersucht. „Die Beschäftigung mit ,Identität' bzw. ,Medien und Identität' hat in den Kultur- und Sozialwissenschaften Hochkonjunktur" - so die Herausgeber des erwähnten Sammelbands in ihrem einleitenden Beitrag, in dem sie die verschiedenen Gründe für diesen "Boom" darlegen. An dieser Stelle soll auf folgende Punkte hingewiesen werden, die in diesem Diskurs (und der damit verbundenen Forschungsarbeit) bislang zu wenig Beachtung finden:

a) Es gibt bislang keine Medienrezeptionsstudie im deutschsprachigen Raum, die entlang eines der o.g. Ansätze die Thematik „Medien und Identitätsbildungsprozesse“ bei Kindern und Jugendlichen aus Migrationskontexten systematisch untersucht hat;

b) Studien und Theoriebildungen zum Thema „Medien und Identität“ in der Medien- und Kommunikationsforschung finden weitgehend unabhängig von Studien und Theoriebildungen zur ldentitätsentwicklung im Bereich der Entwicklungspsychologie statt;

c) Obwohl heute Kinder und Jugendliche Medien zunehmend selbst produktiv nutzen (für Kommunikation und Selbstausdruck), gibt es nach wie vor nur wenige Forschungsarbeiten, die Eigenproduktionen mit Medien als methodische Möglichkeit zur Selbstpräsentation und als Zugang zum Welterleben von Kindern und Jugendlichen nutzen.

Auch die international vergleichende Studie von Livingstone und Bovill (2001) enthält keine Untersuchungen zum Zugang von Kindern und Jugendlichen z.B. zu Kameras und den Produktionsmöglichkeiten mit Multimedia-Computern. Zweifelsohne ist zu beachten, dass es soziokulturelle Unterschiede im Zugang und in der produktiven Nutzung von Foto, Video, Computer und Internet gibt (vgl. insbesondere Studien zum „digital divide" im Bereich der neuen Medien; zusammenfassend: Otto u.a. 2003). Es ist jedoch nicht zu übersehen, dass die aktiv-produktive Nutzung von Medien mit dem Aufkommen des PC und des Internet in informellen Kontexten zugenommen hat und dass seit vielen Jahren medienpädagogische Einrichtungen und Initiativen Formen aktiv-produktiver Medienarbeit in unterschiedlichen pädagogischen Kontexten erfolgreich praktizieren.

Im Bereich der medienpädagogischen Praxisforschung entstanden in Deutschland seit Ende der 80er Jahre mehrere Projekte, die in unterschiedlicher Weise MedienEigenproduktionen von Kindern und Jugendlichen (und damit verbundene Prozesse der Produktion, des Öffentlichmachens und der Interpretation) zum Gegenstand von Forschungsarbeiten machten (zusammenfassend: Niesyto 2001a). Zu nennen ist insbesondere das internationale Projekt „VideoCulture“, das Ende der 90er Jahre in Deutschland, Großbritannien, Tschechien, Ungarn und den USA durchgeführt wurde. Gegenstand war die Produktion und die Interpretation von Videofilmen. Die Filme wurden von 14- bis 19-jährigen Jugendlichen aus verschiedenen soziokulturellen Milieus 
produziert. Entsprechend der Projektvorgabe konnten die Jugendlichen ihre Gefühle, Erfahrungen und Phantasien zu Rahmenthemen wie „Jung sein“ und „Gegensätze ziehen sich an“ mit Bildern und Musik/Tönen auszudrücken, möglichst ohne wortsprachliche Anteile. Die Produktionen entstanden im Rahmen von schulischer und außerschulischer Medienpädagogik. Nach Fertigstellung wurden die Videofilme ausgetauscht und von den beteiligten Gruppen sowie von weiteren Jugendlichen interpretiert. Die am Projekt beteiligten Forscher/innen dokumentierten und analysierten die bei der Symbolproduktion (Workshops) und beim Symbolverstehen (Interpretation der Videofilme) beobachteten Prozesse, Darstellungen und Interpretationsangebote. Das Projekt fragte vor allem nach Formen einer länderübergreifenden, audiovisuellen Symbolsprache sowie nach den Inhalten und Stilen der Symbolverarbeitung, der Symboldarstellung und des Symbolverstehens. Die Ergebnisse, die im Rahmen einer vergleichenden Auswertung von Fallstudien erzielt wurden (Niesyto 2003), konnten auf unterschiedliche Weise die Möglichkeit und Relevanz einer interkulturellen Kommunikation herausarbeiten, die verstärkt ästhetische und symbolische Formen des Selbstausdrucks mit Bildern und Musik integriert. Damit eröffnete das Projekt „VideoCulture“ neue Wege, um einseitig wortlastige Formen der interkulturellen Kommunikation zu überwinden.

\section{Das EU-Forschungsprojekt CHICAM}

„VideoCulture“ war in gewisser Hinsicht ein Vorläuferprojekt für das EU-Projekt „CHICAM - Children in Communication about Migration“1. CHICAM basiert auf Formen einer medienpädagogischen Praxisforschung und verknüpft dies mit der Exploration von Lebenswelten von jungen Migrant/innen. Das Projekt, das von David Buckingham und Liesbeth de Block (London University, Institute of Education) entwickelt wurde, möchte 10- bis 14-jährigen Kindern, die im Kontext von Migration oder Flucht in verschiedenen Ländern Europas leben, die Möglichkeit geben, sich mittels Fotografie, Video und Internet mit der eigenen Lebenssituation auseinanderzusetzen (Peergroup-Beziehungen, Familienbeziehungen, Schule) und Erfahrungen über Ländergrenzen hinweg auszutauschen. Das Projekt verfolgt das Ziel, das Potential neuer Medien für interkulturelle Kommunikation, Reflexion und Integration zu untersuchen. Mit der Vermittlung von Medienkompetenz und Ausdrucks- und Reflexionsfähigkeit sollen die Kinder zu gesellschaftlicher Teilhabe befähigt werden.

In den beteiligten Ländern (Großbritannien/London, Italien/Rom, Niederlande/Utrecht, Schweden/Stockholm, Griechenland/Athen und Deutschland/Ludwigsburg) wurden Gruppen von Kindern, die sogenannten „CHICAM Clubs“ gebildet. Diese ethnisch und geschlechtlich gemischten Gruppen (ca. 8-12 Kinder mit relativ aktueller Migrationserfahrung) arbeiteten über ein Schuljahr hinweg mit digitaler und analoger Fotografie sowie mit Video und hatten die Möglichkeit, mit ihren Partnergruppen über eine speziell eingerichtete Internetplattform zu kommunizieren. Begleitet wurden die Gruppen jeweils von einem/r Medienpädagogen/Medienpädagogin. Eine weitere Person arbeitete in den jeweiligen Clubs, um die Produktions- und Kommunikationsprozesse wissenschaftlich zu begleiten, zu dokumentieren und die Lebenswelten der Kinder zu erforschen. Am EU-Projekt beteiligen sich insgesamt sechs CHICAM-Clubs mit Mädchen und Jungen aus 29 verschiedenen Herkunftsländern.

Der CHICAM Club in Deutschland war in das Ganztagesbetreuungsangebot einer

\footnotetext{
${ }^{1}$ Siehe http://www.chicam.net sowie http://www.ph-ludwigsburg.de/medien1/forsch/chicam.de
} 
Hauptschule im Großraum Stuttgart integriert. Seit Oktober 2002 trafen sich einmal pro Woche acht Kinder aus der Türkei, Kuba, der Dominikanischen Republik, den USA und Tunesien, um Erfahrungen im Umgang mit neuen und alten Medien zu machen. Zu Beginn lag der Schwerpunkt auf dem Gestaltungsbereich Fotografie. Die Kinder konnten sich bei der Produktion von inszenierten Portrait-Aufnahmen, Gruppen- und Ratebildern und im Rahmen von Fotoerkundungen in Ludwigsburg erste technische und ästhetische Basiskompetenzen aneignen. Danach wurde mit dem Medium Video gearbeitet. Es entstanden mehrere kurze Filmproduktionen. Beispielsweise ein Vorstellungsvideo, in dem die einzelnen Clubmitglieder zeigen, was sie mögen und was sie können. In einem Beitrag über Gedanken zum Leben in Deutschland kontrastieren die Kinder Meinungen von Passanten mit ihren eigenen Einstellungen zu Deutschland. Die Kinder lernten ästhetische Gestaltungsformen kennen und entwickelten eine Sensibilität für verschiedene Einstellungen, Perspektiven, das Zusammenspiel von Musik und Bild. Gewalt im Klassenzimmer, Fußballspielen mit einem Monster, Liebesgefühle im Frühling und der klassische Bankraub mit sich anschließender Autoverfolgungsjagd sind Themen, die in verschiedenen Knetanimationsfilmen bearbeitet wurden. Im Bereich Spielfilm entstanden zwei Produktionen zum Thema Freundschaft und Fußball. Aber auch bedeutsame aktuelle Ereignisse wurden von den Kindern im CHICAM Club aufgegriffen und medial umgesetzt. So wurden in Straßeninterviews Stimmen zum Irak-Krieg gesammelt und ein Film gedreht, der sich aus Bildern der Kriegsberichterstattung und aus eigenen Interview-Szenen zusammensetzt.

CHICAM umfasst eine ethnografische und eine medienpädagogische Dimension. Die ethnografische Dimension dient der Erkundung der Lebenswelten der Kinder mittels Medienproduktionen, teilnehmender Beobachtung, Einzel- und Gruppengesprächen. In der medienpädagogischen Dimension werden vor allem Chancen einer audiovisuellen Kommunikation mittels Fotografie, Video und Internet erprobt und erforscht. Projektmitarbeiter/innen erhoben in allen Ländern Daten zu verschiedenen Themen und Lebensbereichen, die für die Kinder relevant sind: Familie, Gleichaltrigengruppen, Schule und Medien. Das Erstellen von Eigenproduktionen und die ethnografische Forschungspraxis sind eng miteinander verbunden. Auf der Basis von Fallstudien entstehen seit Abschluss der Feldphase thematische und summative Analysen: die verschiedenen Daten, die ihm Rahmen der CHICAM-Clubs erhoben wurden, werden aufbereitet, entlang der Projektleitfragen analysiert, unter den Forschern und Forscherinnen der sechs beteiligten Länder verglichen und diskutiert. Abschlussberichte entlang einzelner Themenbereiche werden ab Sommer 2004 erscheinen. Einige Partner verfügen über spezielle Erfahrungen im Bereich Migrationsarbeit und -forschung, andere dagegen mehr im Bereich Medienpädagogik und forschung. Die Abteilung Medienpädagogik an der $\mathrm{PH}$ Ludwigsburg ist zusammen mit dem Institute of Education, University of London, für den Bereich der visuellen Kommunikation zuständig. Beide Partner verfügen über Vorerfahrungen aus dem bereits erwähnten Projekt „VideoCulture“. Im Projekt CHICAM sollen die Beobachtungen aus „VideoCulture“ anhand einer jüngeren Altersgruppe erweitert werden. Mit dieser Grundlagenarbeit möchten die Forschungsgruppen Konzepte für zeitgemäße Formen von interkultureller Medienarbeit und „Image Education“ entwikkeln.

Obgleich spezifische Fragen zu Identitätsbildungsprozessen bei den Kindern/Jugendlichen, die in den CHICAM-Clubs mitwirkten, nicht expliziter Gegenstand der Forschungsarbeit waren, möchten wir im Folgenden versuchen, einige Beobachtungen und Erfahrungswerte aus der CHICAM-Projektarbeit in Deutschland unter diesem Gesichtspunkt vorzustellen. Dabei zu beachten, dass Identitätsbildungsprozesse 
bei 10-15Jährigen eng mit altersspezifischen Entwicklungsaufgaben in der Übergangsphase von Kindheit zu Jugend zusammenhängen, bei der man nicht von existierenden „individuellen Rahmenkonzepten“ einer Person sprechen kann, innerhalb derer Erfahrungen interpretiert und zur Basis für alltägliche Identitätsarbeit gemacht werden. Dennoch stellt sich die Frage, wie Kinder/Jugendliche in dieser Altersphase die Vielzahl von Eindrücken im Spannungsfeld der verschiedenen Sozialisationsfelder verarbeiten und wie sie altersgemäß ein „Gefühl von Identität“ (Keupp 1998) entwickeln. In Anlehnung an Keupp verstehen wir Identitätsarbeit als einen evaluativen Prozeß, der selbstreflexive Fähigkeiten (Vorstellungen über das Selbst, die Welt der Objekte und der sozialen Situationen) voraussetzt. Diese selbstreflexiven Fähigkeiten hängen nicht nur von altersbedingten Fähigkeiten (z.B. kognitiver Entwicklungsstand, Perspektivenwechsel etc.), sondern auch von sozialen und kulturellen Ressourcen ab, die Kindern und Jugendlichen in unterschiedlicher Weise zur Verfügung stehen. Der Umgang mit Gefühlen und Erfahrungen der Ambivalenz ist dabei eine Aufgabe, die sich in allen Altersphasen stellt. Die Frage ist, welche Orientierungsangebote Kinder und Jugendlich aus unterschiedlichen Erfahrungsfeldern aufgreifen und wie sie diese Angebote nutzen und verarbeiten, um jeweils alters- und situationsspezifische „Passungen“ und Handlungsfähigkeiten für sich zu erreichen. Im Unterschied zu kognitiv verengten Konzepten gehen wir davon aus, dass emotionale Orientierungen und Verarbeitungsprozesse für die Herausbildung dieser Handlungsfähigkeiten sehr wichtig sind.

Der aktiv-produktive Umgang mit Medien, das Erstellen von Eigenproduktionen mit Medien bietet - so unsere Annahme -, Kindern/Jugendlichen eine wichtige Möglichkeit, in spielerischer und anschaulicher Form eigene Gefühle, Bedürfnisse und Phantasien auszudrücken. Der Schritt von der Medienrezeption zur Medienproduktion eröffnet Möglichkeiten für Selbstausdruck, ästhetisch-symbolisches Probehandeln und Kommunikation, integriert emotional-anschauliche und planerisch-reflexive Dimensionen und bietet einer explorativ orientierten Forschung Einblicke in Prozesse der symbolischen Verarbeitung, Darstellung und Interpretation lebensweltlicher Erfahrungen (Niesyto 2001b).

\section{Medien im Migrationskontext ${ }^{2}$}

In Bezug auf Musikpräferenzen schöpfen die CHICAM-Clubmitglieder aus einem breiten musikkulturellen Ressourcenspektrum. Musik aus dem Herkunftsland (türkische, kurdische, lateinamerikanische, tunesische Musik) global vermarktete, meist englischsprachig Musik (z.B. Shakira, Eminem, Jenifer Lopez, Avril Lavinge) und zum Teil auch deutschsprachige Musik. Ähnlich ist auch der Bereich Fernsehnutzung: sowohl muttersprachliche (türkisch, kurdisch, spanisch, arabisch) als auch deutschsprachige Fernsehprogramme werden genutzt. Es fällt auf, dass Privatsender wie RTL2 und Pro7 besonders beliebt sind. Auch Sender mit Musikvideos (MTV, VIVA) sind populär.

Es scheint so, als würden die Kinder/Jugendlichen über ihre medialen Präferenzen symbolisch Nähe zu verschiedenen kulturellen Kontexten herstellen und ausbalancieren (z.B. türkische Musik und global zugängliche Musik wie z.B. Hip-Hop).

Musikpräferenzen wurden auch immer wieder im Club selbst deutlich. Es war üblich, dass einige der Mitglieder schon vor Beginn des Treffens kamen und ihre Musik mit-

\footnotetext{
${ }^{2}$ Das Verhältnis von Migration und Medien kann auf verschiedenen Ebenen untersucht werden:

a) Wie werden Migranten in den Medien dargestellt, wie sind sie repräsentiert? (vgl. z.B. Schorb 2003)

b) Wie nutzen Migranten Medien? (vgl. z.B. Weiß und Trebbe 2001)

c) In welcher Weise produzieren Migranten Medien (aktive Medienarbeit, Radio- und Fernsehsender)?
} 
brachten, sich unterhielten und zum Teil tanzten. Dabei kam es immer wieder zu Situationen, in denen ausgehandelt werden musste, wessen Musik gespielt werden soll. Diese Auseinandersetzungen und Aushandlungsprozesse wurden jedoch nie auf nationaler, sondern eher auf persönlicher Ebene geführt.

Deutschsprachiges Fernsehen wird - ebenso wie Musik und Hörspielkassetten - genutzt, um die Entwicklung von Sprachkompetenzen zu unterstützen. Melek, ein Mädchen aus der Türkei nutzte insbesondere Kinderprogramme um Deutsch zu lernen und schaute mit ihrem kleinen Bruder zusammen: „Ich will lernen von Deutsch, weil meine Bruder immer kuckt, ich kucke auch." Interessant an dieser Nutzungsweise ist die Tatsache, dass zusätzlich zur Schule selbstgewählte eher informelle Aneignungssituationen geschaffen werden, die auch eine soziale und familiäre Dimension enthalten (gemeinsame Medienerfahrungen mit dem jüngeren Geschwisterteil). Die Motivation Deutsch zu lernen war bei den Clubmitgliedern insgesamt sehr hoch. Ein Junge befürchtete sogar, dass durch einen Besuch in der Türkei in den Sommerferien Lernfortschritte rückgängiggemacht werden könnten.

Auch Serife, die keine jüngeren Geschwister hat, nutzt deutschsprachige Programme und Kinderfilme: "...kuck ich auch den Deutschen Kanal, weil will ich auch Deutsch lernen, kuck ich auch Kinderfilm."

Während deutschsprachige Sender die Entwicklung der neuen Sprache begünstigen und potenziell auch Gemeinsamkeit stiften, können muttersprachliche Programme, die über Kabel- und Satellitenfernsehen empfangen werden, eine Form des Kontakthaltens zur Herkunftskultur und zur Muttersprache ermöglichen. ${ }^{3}$

Für Hakans Vater scheint diese Dimension des Kontakthaltens keine Bedeutung zu haben. Er möchte, dass sein Sohn deutschsprachige Programme schaut und keine türkischsprachigen, um die Entwicklung seiner deutschen Sprachkompetenzen nicht einzuschränken: „Ich muss nicht so türkische Programme schauen nicht immer Deutsche.“ In einem anderen Zusammenhang berichtet Hakan, dass ihm beim Telefonieren mit seiner in der Türkei lebenden Mutter manche türkischen Wörter nicht mehr einfallen:

Hakan: „Ich spreche bisschen Deutsch, weil ich hab' Türkisch vergessen. Ich spreche mit meine Mutter. Ich denke immer 'was sag ich, was sag ich', ich denke Deutsch aber ich sage meiner Mutter Deutsch meine Mutter sagt 'was ist das' ich denke 'was ist das was ist das auf türkisch' und so."

Über das Betrachten persönlicher Fotografien aus dem Herkunftsland können Erinnerungen an Situationen und abwesende Personen wachgerufen werden. In diesem Sinne könnte auch von einer Kontakthaltefunktion gesprochen werden. Die Bereitschaft der Clubmitglieder, Einblicke in diese Fotografien zu geben war unterschiedlich groß.

Das Interesse an internationaler Musik verbindet die Clubmitglieder zum Teil untereinander, zum Teil aber auch mit anderen Kinder und Jugendlichen an ihrer Schule, in Deutschland allgemein und in vielen anderen globalisierten Ländern.

\footnotetext{
${ }^{3}$ Güntürk (1999) nennt verschiedene Funktionen muttersprachlicher Medien:

- Über muttersprachliche Medienangebote Informationen über das Ursprungsland erhalten, die die Medien im Einwanderungsland nicht anbieten

- Durch muttersprachliche Medienangebote Kontakt zum Ursprungsland und zur Muttersprache halten „Brücke zur Heimat“

- muttersprachliche Medien als Sprachrohr der Migranten

- muttersprachliche Medienangebote als Beitrag zur Herausbildung einer etnisch-kulturellen Identität
} 
Hip-Hop als globale Jugendkultur ist ein Beispiel für gemeinsam geteiltes popkulturelles Kapital (Fiske), das nicht nur innerhalb des Clubs ein verbindendes Element darstellen kann, sondern auch zwischen den Clubs (vgl. Hip-Hop-Videoeigenproduktionen in verschiedenen CHICAM-Clubs, so in London: „A place to be“, Niederlande: „Abdelmajid's Rap“, Schweden: Coverversion von 50 Cent: In da Club), Eminem im Vorstellungsvideo Italien). ${ }^{4}$ Die Teilhabe an medialen Diskursen kann als partielle Inklusion verstanden werden. Bilder von Männern und Frauen in den Medien stellen potenzielle Orientierungsangebote in bezug auf Geschlechtermodelle dar. Die Symbolwelt des Hip-Hop war für ein Clubmitglied aus der Türkei als Modell für "Coolness“ attraktiv.

Das Interesse an YU-GI-OH-Spielkarten, das in verschiedenen Clubs vorhanden war (Griechenland), stellt ein weiteres Beispiel für interkulturell verbindendes Medienhandeln dar. Fernando konnte sich über das YU-GI-OH-Spiel einen Freundeskreis in seiner Nachbarschaft erschließen. Interessant wären die verschiedenen Umgangsweisen und Bedeutungen und Regeln zu untersuchen, die den Spielkarten in verschiedenen kulturellen Kontexten gegeben werden.

\section{Kulturaspekte und ihre unterschiedlichen Bewertungen}

In Abhängigkeit zu kulturellen Wertigkeiten (Symbolhierarchien), die unter anderem auch über Medien kommuniziert und ausgehandelt werden, erfahren Kinder/Jugendliche mit Migrationshintergrund Anerkennung oder Ablehnung für ihre kulturellen Besonderheiten (z.B. Ablehnung bzw. Mitleid für Kopftuch-Tragen und Anerkennung für Salsa-Tanzen).

Taskania mag Musik aus der Dominikanischen Republik und anderen Süd- und Mittelamerikanischen Ländern (Salsa, Merengue, Batschata) und sie tanzt auch sehr gerne. Sie erfährt Anerkennung für ihre Kompetenzen im Bereich lateinamerikanische Tänze. Lehrerinnen baten sie darum, ihnen Tanzschritte beizubringen und es wird ihr ein Expertinnenstatus für diese als positiv bewerteten Kulturelemente zugeschrieben (z.T. auch positiv gemeinte Stereotypisierungen wie „Sie hat das Tanzen im Blut“).

Aufgrund von Popularisierung, globaler Verbreitung und Vermarktung erfahren in Deutschland (und vielen anderen westlichen Ländern) Musik und Tanzstile aus Mittelund Südamerika nicht nur in speziellen Fankulturen positive Urteile.

Türkische Mädchen aus dem CHICAM-Club und auch Freundinnen von innen aus der Klasse erhielten von Lehrerinnen und Mitschülern negative Rückmeldungen in bezug auf ihre Kopftücher. (Im Vorstellungsvideo wird ein Mädchen mit Kopftuch provozierend gefragt, ob sie Haare hat. Sie antwortet: „Ja”.)

In der Bundesrepublik Deutschland wird das Kopftuch oft als Symbol der rückständigen Traditionsverhaftung und der männlichen Unterdrückung türkischstämmiger Frauen gedeutet, weniger als Ausdruck von Stolz und aktiver Selbstzuordnung.

Zwei der kopftuchtragenden Mädchen im Club - Fatma und Serife - trugen das Kopftuch nicht aus eigener freier Entscheidung. Serife hatte eine zeitlang stark unter dem Druck zu leiden, der ihr großer Bruder auf sie ausübte, indem er den symbolischen Raum ihres Körpers zu reglementieren versuchte (vgl. Schiffauer et al. 2002, 326-331). Serifes Fall könnte als Bestätigung der oben genannten negativen Kopftuchdeutung verstanden werden (das Kopftuch als männliche Unterdrückung).

\footnotetext{
${ }^{4}$ Kulturübergreifende Medienerfahrungen können auch als geteilter Raum oder als „third culture“ (Featherstone 1995) verstanden werden.
} 
Der folgende Ausschnitt einer Gruppendiskussion im Club verdeutlicht, dass negative Bewertungen sowohl durch Lehrerinnen als auch durch Mitschüler vorgenommen wurden:

Hakan: Ja, Frau Wönsch hat gesagt, ähh, du musst Kopftuch machen oder dein Eltern sagt so.

Fatma: Frau Wönsch und Frau Güd hat gesagt äh du musst Kopf nicht mach Kopftuch macht nicht (andere sprechen durcheinander) und dann Serife Gonca auch Kopftuch machen und dann ähh und dann Elte Elternabend kommt und dann ähh macht nicht Kopftuch Frau Wönsch ist ,schon gut schon gut' und Frau Güd hat gesagt ,schon gut schon gut Kopftuch nicht schön' warum nicht schön Kopftuch?

Hakan: Wie eine Hexe, die Kopftuch ist scheiße, ohne Haare guck mal ohne Haare was soll äh schauen.

Björn: Also, du, du findest es nicht gut wenn jemand Kopftuch trägt warum, weil man die Haare nicht sieht und wie findest du das mit dem Kopftuch?

Hakan: Weil so ist besser

Fatma: Ich will nicht Kopftuch machen aber meine Mutter und mein Vater hat gesagt das machen.

Björn: Ach so, also es war nicht deine Entscheidung sondern deine Eltern. Du würdest lieber kein Kopftuch haben.

Hakan: Meine Schwester...

Fatma: Ich will, ich will nicht.

Hakan: ...meine Schwester ein Monat hat ein Kopftuch gemacht, Frau Güd hat gesagt ,ah Gonca schlecht diese Kopftuch' und dann meine Schwester hat meine Schwester hat weggemacht die Kopftuch und Frau Güd hat gesagt ,Gonca jetzt ist schön du bist so schön.'

Peter: $\mathrm{mhm}$

Björn: Mhm, und, ah warum hat deine Schwester ein Kopftuch ah angezogen?

Hakan: Meine Schwester will so, vielleicht Kopftuch, vielleicht keine. Meine Vater sagt nicht, du musst Kopftuch machen'.

\section{Medienbildung}

Ein Projektziel auf der praktischen und politischen Ebene war es, Integrations- und Entwicklungspotenziale von praktischer Medienarbeit in Migrationskontexten zu untersuchen und medienpädagogische Konzepte für die Zielgruppe Kinder/Jugendliche mit Migrationshintergrund zu erproben und weiterzuentwickeln. Im Rahmen der Vorarbeiten wurde deutlich, dass in diesem Bereich noch relativ großer Entwicklungsbedarf besteht.

Eine Förderung von Kindern/Jugendlichen mit Migrationshintergrund im produktiven Umgang mit Medien erscheint insbesondere aufgrund folgender Potentiale wichtig:

- im Umgang mit Medien kann Selbstwirksamkeit erfahren und Selbstwertgefühl entwickelt werden (Menschen auf der Straße ansprechen; mit dem Medium Video neue Räume erkunden);

- aktive Medienarbeit ermöglicht Reflexions- und Ausdrucksmöglichkeiten, über Medienarbeit können neue Perspektiven eingenommen werden;

- über Medienarbeit kann Kontakt zwischen Migranten und Nicht-Migranten entstehen bzw. hergestellt werden (z.B. Interviews auf der Straße, Präsentation von Medienprodukten); 
- Ausdruck mit Medien kann sprachliche Defizite kompensieren, dabei verbindet Medienarbeit Medienkompetenz und Sprachkompetenz.

Im Zusammenhang mit Lernfortschritten im Spracherwerb kommt ein Lehrer der CHICAM-Clubmitglieder zu folgender Einschätzung:

„Das ganze war 'ne Belebung für die Klasse für die Gruppe auch und hat hineingewirkt in die Klasse hinein auch ... des isch was Außergewöhnliches gewese' und kommt auch nicht wieder so schnell." [...]

„Ich glaube auch gerade durch des CHICAM muss ich ehrlich sagen, ja, des hat des is, war 'ne sehr gute Sache, die wirklich motiviert hat und die den Schülern auch geholfen hat gerade mit ihnen zusammen dann in der Gruppe etwas zu erleben und dabei auch zu gewinnen an Sprachwert, an Sprachqualität."

Frau Wönsch, die Klassenlehrerin ergänzt: „Weil der CHICAM-Club der setzt sich ja doch aus verschiedenen Nationen zusammen, da müssen sie einfach Deutsch reden, ja, und des geht dann automatisch."

Diese Aussagen sowie die Beobachtungen im CHICAM-Club über ein Jahr hinweg machen deutlich, dass der Club einen Beitrag zur Sprachentwicklung leisten konnte, auch wenn dieser Aspekt gar nicht explizit Teil der Intentionen war. Sprachliche Fortschritte konnten sich wiederum positiv auf die Verstehensmöglichkeiten von medienpädagogischen Inputs auswirken, was die Aneignung von Medienkompetenzen erleichtert. Möglicherweise ist gerade der Umgang mit visuellen und audiovisuellen Medien, an dem die wortsprachliche Kommunikation anknüpfen kann, besonders zur Sprachentwicklung geeignet.

Bei der Entwicklung und Anwendung praktischer Konzepte interkultureller Medienarbeit sind die Dimensionen Subjektorientierung, Anschaulichkeit/Visualisierung, Strukturierung und Minimierung von Frustrationserfahrungen/Maximierung von Erfolgserlebnissen von großer Bedeutung. Die im Folgenden dargestellten Aspekte basieren weitgehend auf Maurer (2003).

Subjektorientierung kann bedeuten, Impulse und Ideen der beteiligten aufzugreifen: Nach Beginn des Golfkriegs im März 2003 wurde im Club über das Thema gesprochen und die Kinder/Jugendlichen äußerten das Bedürfnis, in der Innenstadt Passanten zum Thema „Krieg“ mit der Kamera zu befragen.

Das Aufgreifen lebensweltlicher Bezüge kann auch bedeuten, zur Veranschaulichung medienästhetischer Gestaltungsmöglichkeiten Videoclips von Musikstücken zu zeigen, die die Kinder und Jugendlichen mögen.

Anschaulichkeit/Visualisierung kann bedeuten, den Umgang mit Medien nicht nur verbal zu erklären, sondern spezielles visuelles Material zu zeigen. So veranschaulichte der Medienpädagoge Björn Maurer den digitalen Filmschnitt, indem er Filmstreifen aus Papier, die mit Bildern aus dem selbst gefilmten Material der Clubmitglieder bedruckt waren, mit der Schere zerschneiden und neu arrangieren ließ. 

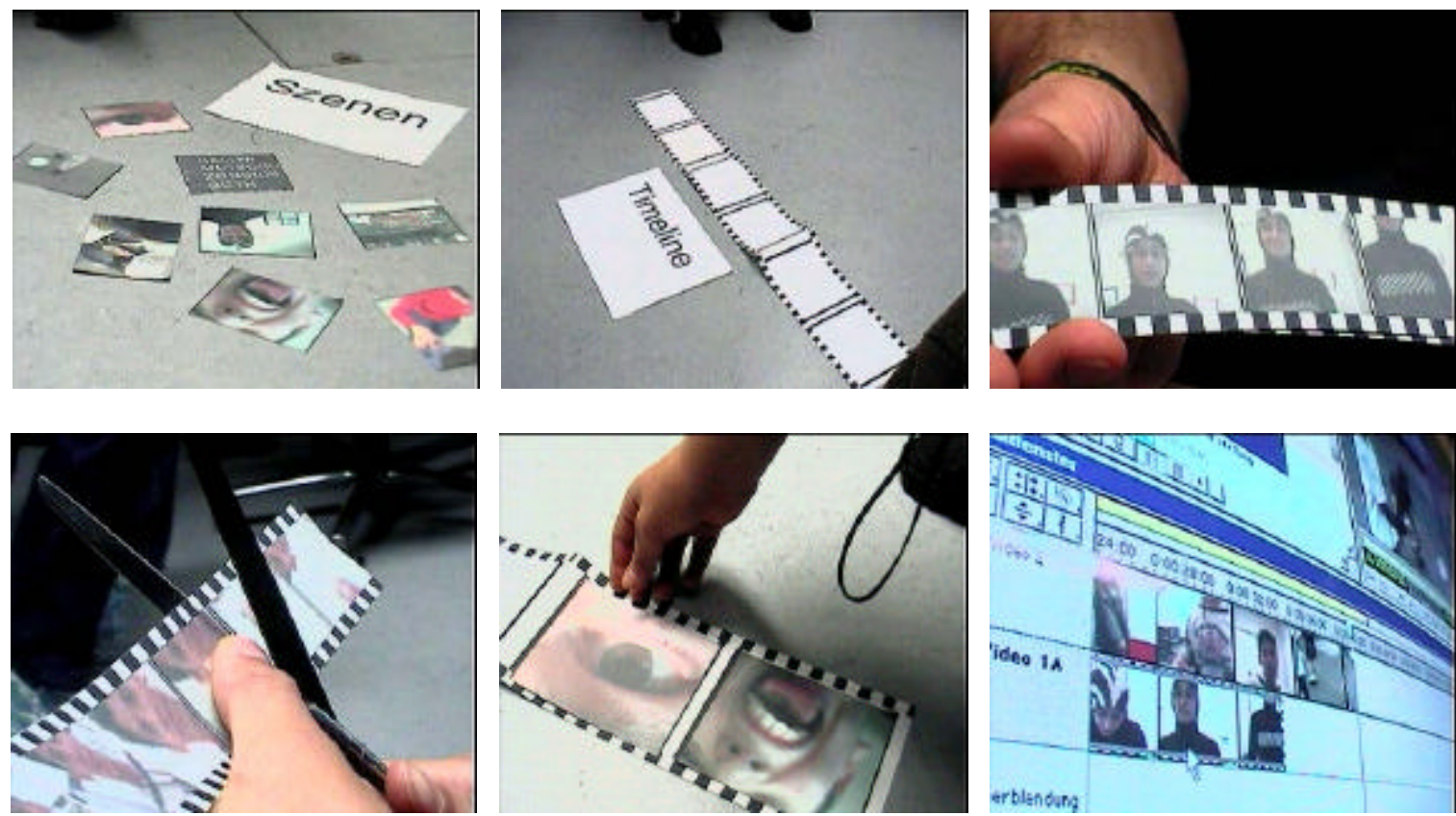

Veranschaulichung des digitalen Videoschnitts anhand von speziellem Material

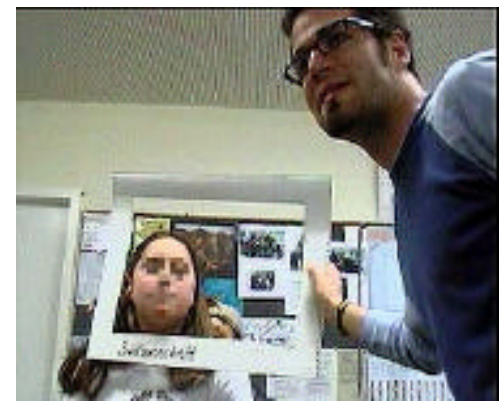

Veranschaulichung der Ausschnittgestaltung

Strukturierung kann bedeuten, Lernziele auf konkrete Kameraübungen herunterzubrechen und auf einem Papier genau zu erklären und zu visualisieren.
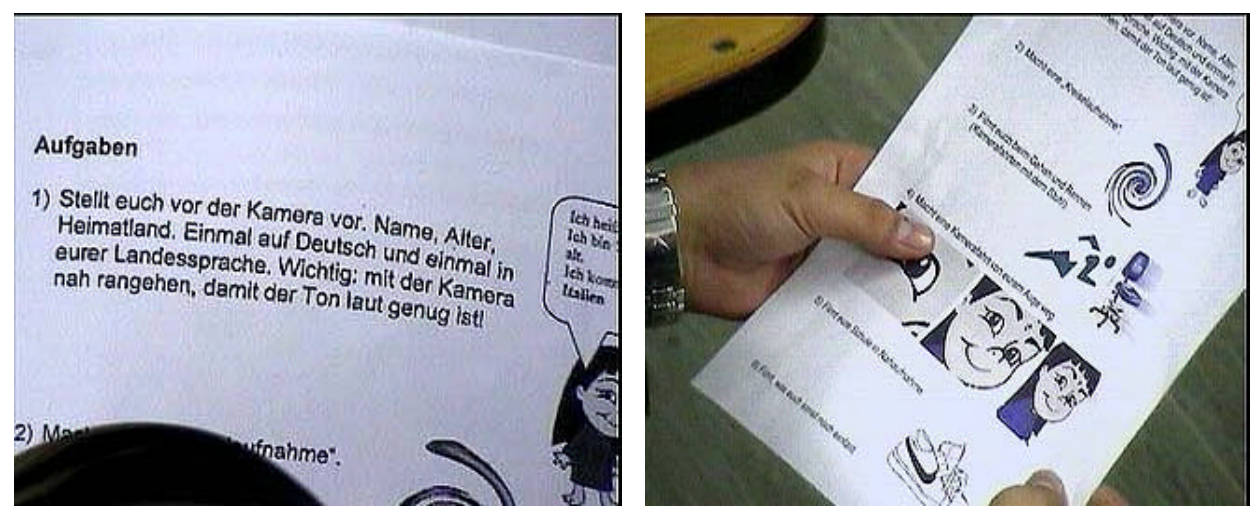

Aufgabenblatt mit Erklärungen und Visualisierungen 
Minimierung von Frustrationserfahrungen ${ }^{5}$ / Maximierung von Erfolgserlebnissen kann bedeuten,

- beim digitalen Videoschnitt am Computer die zu bearbeitenden Szenen bereits vorher auszuwählen und zu digitalisieren;

- den Kindern/Jugendlichen die Möglichkeit zu geben, an ungewöhnlichen und interessanten Orten zu filmen, an denen sie sich sonst nicht aufhalten und die sie nur wegen der Kamera und des Medienprojekts betreten dürfen;
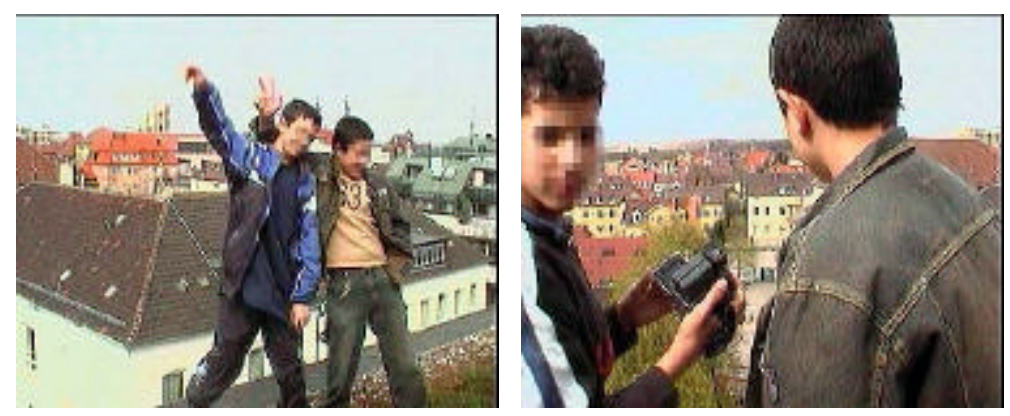

Clubmitglieder filmen ihre Schule vom Dach eines Nachbargebäudes aus

- motivierende Clips aus dem Material der Kinder zu schneiden um die Möglichkeiten des Schnitts und die Wirkungskraft der Aufnahmen zu veranschaulichen (vgl. Holzwarth/Maurer 2003);

- den Kindern/Jugendlichen eine Videokamera mit eingestelltem Spezialeffekt (z.B. Verwischungseffekt oder Stroboskop) zu geben, damit sie ungewöhnliche ästhetische Erfahrungen machen können (vgl. Holzwarth/Maurer 2003).
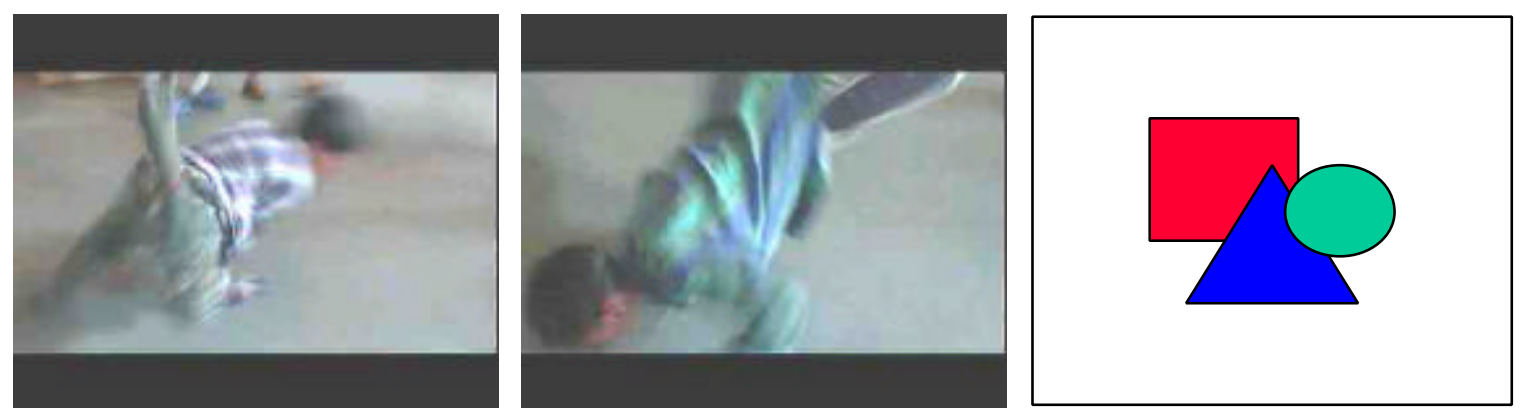

Standbilder von einer Breakdance-Aufnahme mit eingestelltem Verwischungseffekt
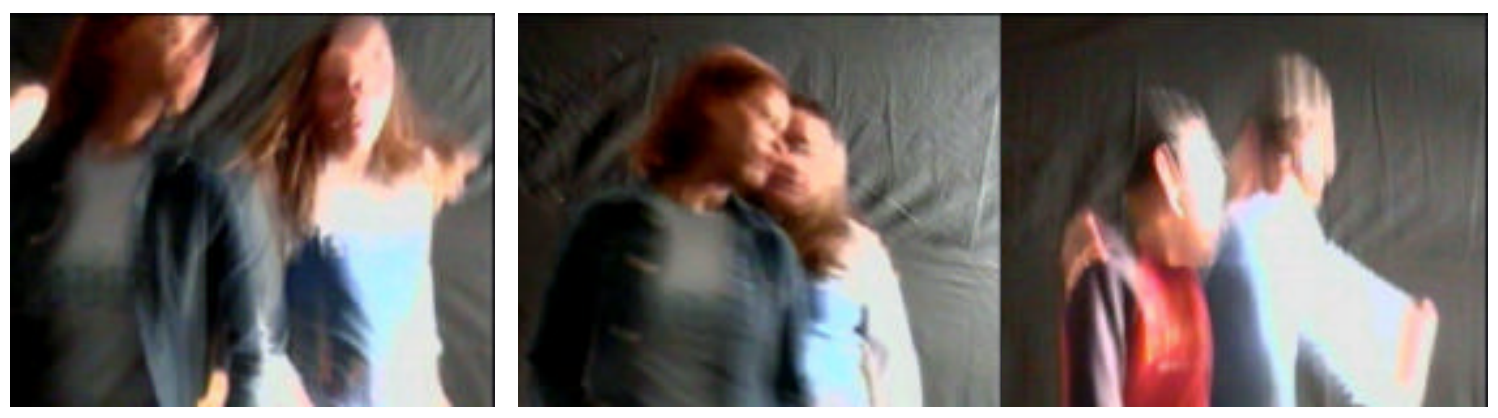

Standbilder von einer Tanzaufnahme mit eingestelltem Verwischungseffekt

\footnotetext{
${ }^{5}$ Minimierung von Frustrationserfahrungen muss nicht bedeuten, dass Frustrationstoleranz nicht langfristig als Lernziel angestrebt werden kann bzw. angestrebt werden sollte;
} 
- dafür zu sorgen, dass die Produzierenden auch Rückmeldungen zu ihrer Arbeit von einem Publikum bekommen können (vgl. Buckingham/Harvery 2003; Holzwarth 2003).

Medienpädagogische Angebote, die auch die körperliche Dimension berücksichtigten waren besonders beliebt, z.B. zu selbst mitgebrachter Musik zu tanzen bzw. vor der Kamera zu raufen und zu kämpfen, Fußball zu spielen oder auch mit der Kamera auf die Straße zu gehen und Passanten zu befragen. In Bezug auf die medienpädagogische Begleitung wurde auch das Bedürfnis nach kürzeren verbalen Erklärungen geäuBert.

Arbeitsformen, die eine relativ schnelle Rückmeldung ermöglichen, waren für die meisten Kinder/Jugendlichen attraktiver als Formate, bei denen das fertige Produkt erst nach längerer Zeit und größerem Aufwand entsteht. So ermöglichen z.B. Knetanimationen ein fertiges Produkt in einer überschaubaren Zeit (ca. 2-3 Stunden).

Der Aspekt Betreuung spielt für die Motivation eine besondere Rolle. Je weniger Aufmerksamkeit mit anderen geteilt werden muss, desto höher die Motivation. Der Animationsfilm „At the beach“ entstand während eines sehr produktiven und arbeitsintensiven Treffens. Mustafa, ein Junge aus Tunesien, war in dieser Einzelbetreuungssituation auffällig ruhig, motiviert und diszipliniert. Dieses konzentrierte Verhalten stand in einem starken Kontrast zu auffälligen, non-konformen Verhaltensweisen, die er in machen Gruppensituationen gezeigt hatte. Es ist anzunehmen, dass Mustafa in der Einzelbetreuungssituation der Kompaktphase keine Notwendigkeit sah, Aufmerksamkeit auf sich zu lenken.

Dieses Beispiel zeigt auch, dass nicht nur bestimmte Media-models Bedürfnissen von Kindern und Jugendlichen mehr oder weniger gerecht werden, sondern dass auch Sozial- und Gruppenformen bestimmte Prozesse ermöglichen oder verhindern können.

Für Jugendliche wie Mustafa wäre es möglicherweise wichtig (soweit die finanziellen und personellen Ressourcen es erlauben), zunächst Erfolgserlebnisse in Einzelbetreuungssituationen zu ermöglichen, um dann - nach und nach - zu Gruppensituationen überzugehen. ${ }^{6}$

Die folgende Tabelle vermittelt einen Einblick in die Erfahrungen, die in der Praxisphase des Projekts CHICAM mit konkreten Media-models gesammelt werden konnten.

Es stellt sich die Frage, welche Möglichkeiten des Selbstausdrucks bestimmte Formate für Kinder/Jugendliche mit Migrationserfahrung eröffnet werden können und wo jeweils die Grenzen liegen.

\begin{tabular}{|l|l|l|}
\hline $\begin{array}{l}\text { Media- } \\
\text { models } \\
\text { oder For- } \\
\text { mate }\end{array}$ & Möglichkeiten & Grenzen \\
\hline $\begin{array}{l}\text { Knet- } \\
\text { Animation }\end{array}$ & $\begin{array}{l}\text { - innerhalb geringer Zeit (ca. 2-3 Stunden) } \\
\text { können gute Ergebnisse erzielt werden } \\
\text { - Zwischenergebnis (animierte Objekte an- } \\
\text { schauen) ist motivierend }\end{array}$ & $\begin{array}{l}\text { - keine eigene körperliche Inszenierung } \\
\text { möglich } \\
\text { - Kneten erfordert Geduld } \\
\text { - ist nur für kleine Gruppen geeignet } \\
\text { - ist relativ aufwendig (Technik, Betreuung) }\end{array}$ \\
\hline
\end{tabular}

\footnotetext{
${ }^{6}$ Ein problematischer Punkt besteht darin, dass in vielen sozialen Kontexten (z.B. Schulklasse, MedienAG, Jugendhaus) intensive Betreuung besonders wichtig wäre, aus finanziellen und personellen Gründen aber nicht möglich ist.
} 


\begin{tabular}{|c|c|c|}
\hline & \begin{tabular}{|l|} 
und Figuren gearbeitet (es ist keine große \\
und aufwendige Story notwendig) \\
- Formalästhetische Aspekte können ver- \\
mittelt werden (z.B. Perspektive verändern) \\
- Imaginäres kann sehr gut dargestellt wer- \\
den (z.B. auch Dinge, die nicht in der Wirk- \\
lichkeit real existieren (z.B. Monster)) \\
- Knetanimationen wirken meistens sehr in- \\
teressant, auch wenn mit geringen Perfekti- \\
onsansprüchen gearbeitet wurde (z.B. Figu- \\
ren mit ruckartigen Bewegungen)
\end{tabular} & \\
\hline Spielfilm & $\begin{array}{l}\text { - es ist möglich, lebensweltbezogene Ge- } \\
\text { schichten zu entwickeln } \\
\text { - beim Schnitt gibt die narrative Struktur vor, } \\
\text { welche Bildsequenzen in welcher Reihen- } \\
\text { folge ins Storyboard eingefügt werden } \\
\text { - alternativ zu verbalorientierten Ansätzen } \\
\text { kann auf der Grundlage eines groben Kon- } \\
\text { zepts spontan und intuitiv gearbeitet werden } \\
\text { (Inspirationen, die vom Drehort ausgehen } \\
\text { aufgreifen) } \\
\text { - Bedürfnis nach ,Action' und Bewegung } \\
\text { kann ausgelebt werden } \\
\text { - die Attraktivität, die von der Welt des Films } \\
\text { ausgeht kann genutzt werden }\end{array}$ & $\begin{array}{l}\text { - Als Genre generell nicht problematisch, } \\
\text { problematisch wäre nur ein zu planerischer } \\
\text { und sprachzentrierter Zugang } \\
\text { - Schnitt ist Problematisch wegen gering } \\
\text { ausgeprägter Frustrationstoleranz (Er- } \\
\text { folgserlebnisse ergeben sich erst nach } \\
\text { längerer Zeit) } \\
\text { - es sind nur relativ kurze Filme möglich } \\
\text { (Aufwand) } \\
\text { - darstellerische Interessen und Kompe- } \\
\text { tenzen verlangt } \\
\text { - Geschichten müssen entwickelt werden } \\
\text { (Individualbetreuung) }\end{array}$ \\
\hline Videoclip & \begin{tabular}{|l|} 
- Anknüpfen an Medienvorlieben und Wahr- \\
nehmungsgewohnheiten möglich \\
- Bedürfnisse nach Bewegung und Selbstin- \\
szenierung können aufgegriffen werden \\
\end{tabular} & $\begin{array}{l}\text { - Auswahl von verschiedenen Sequenzen } \\
\text { kann überfordernd sein } \\
\text { - Schnitt ist problematisch (s. Spielfilm) }\end{array}$ \\
\hline $\begin{array}{l}\text { Befragung } \\
\text { auf der } \\
\text { Straße }\end{array}$ & $\begin{array}{l}\text { - Kinder/Jugendliche konnten die Rolle des } \\
\text { Interviewers kennen lernen, (was von Vorteil } \\
\text { ist, wenn sie im Rahmen von Praxisfor- } \\
\text { schung auch selbst interviewt werden) } \\
\text { - Kinder/Jugendliche können sich als aktiv } \\
\text { handelnde Subjekte erleben } \\
\text { - Teilnehmende haben die Möglichkeit, sich } \\
\text { einer Herausforderung zu stellen } \\
\text { - es können sich unter Umständen interes- } \\
\text { sante Gespräche zwischen Interviewern und } \\
\text { Interviewten entwickeln (z.T. Rückfragen an } \\
\text { die Interviewer) } \\
\text { - wahrnehmungsleitende und fokussierende } \\
\text { Karten zur Reflexion der Aufnahmen kön- } \\
\text { nen eine sinnvolle Hilfe darstellen } \\
\text { - die Kamera kann Mut machen und die ei- } \\
\text { gene Position stärken (Macht) } \\
\text { - Kontakt zur Mehrheitsbevölkerung möglich } \\
\text { (und durch Kamera legitimiert) } \\
\text { - auch wenn nicht interviewt wurde, konnten } \\
\text { Erfahrungen } \\
\text { beim Filmen gesammelt werden } \\
\text { - es kann auch in der Muttersprache inter- } \\
\text { viewt werden (z.B. Türkisch) } \\
\text { - Herausgehen und Raum aneignen (Bewe- } \\
\text { gung) }\end{array}$ & $\begin{array}{l}\text { - nicht alle haben sich getraut, Leute zu } \\
\text { fragen, nicht alle konnten positive Erfah- } \\
\text { rungen machen } \\
\text { - Sprachliche Grenzen der Kin- } \\
\text { der/Jugendlichen können deutlich werden } \\
\text { - Befragung für manche hohe Hemm- } \\
\text { schwelle } \\
\text { - es kann zu Frustrationserfahrungen bei } \\
\text { Abweisung (Gesprächsverweigerung) } \\
\text { kommen }\end{array}$ \\
\hline $\begin{array}{l}\text { Einweg- } \\
\text { kamera }\end{array}$ & \begin{tabular}{|l|} 
- Kinder/Jugendliche können quasi autonom \\
über das Medium verfügen und unabhängig \\
von pädagogischen Kontexten Bilder ihrer \\
Lebenswelt erzeugen (Interessant für die \\
Forschung ist die Möglichkeit, Zugänge zu \\
ansonsten unzugänglichen lebensweltlichen \\
\end{tabular} & $\begin{array}{l}\text { - Prozesse der Kompetenzaneignung kön- } \\
\text { nen nicht direkt durch medienpädagogi- } \\
\text { sche Beratung unterstützt werden }\end{array}$ \\
\hline
\end{tabular}




\begin{tabular}{|l|l|l|}
\hline & $\begin{array}{l}\text { Feldern zu finden (z.B. Familie). Fotos eig- } \\
\text { nen sich sehr gut als Grundlage für Inter- } \\
\text { views, insbesondere dann, wenn die } \\
\text { sprachlichen Ausdruckskompetenzen noch } \\
\text { eingeschränkt sind) }\end{array}$ & \\
\hline Digital- \\
fotografie & $\begin{array}{l}\text {-,neue“ Technik als Motivation } \\
\text { - Bild kann gleich angeschaut werden (keine } \\
\text { Entwicklung notwendig) } \\
\text { - Bilder können einfach vergrößert und aus- } \\
\text { gesellt werden (Aspekt Öffentlichkeit) }\end{array}$ & $\begin{array}{l}\text { - begrenzte Anzahl an Kameras kann zu } \\
\text { Aushangsschwierigkeiten führen }\end{array}$ \\
\hline
\end{tabular}

Das folgende Fallbeispiel verdeutlicht, wie durch aktive Medienarbeit Kommunikation, Reflexion und Weiterentwicklung angestoßen werden kann:

Neben Knetanimationen (die keiner Nachproduktion bedürfen) und kleinen narrativen Filmen war bei den Kindern/Jugendlichen eine reportageartige Form beliebt, bei der sie mit der Videokamera auf die Straße gehen und Passanten befragen konnten.

Das Video "Deutschland positiv/negativ" besteht aus Reaktionen von allochtonen und authochtonen Erwachsenen und Jugendlichen auf die Fragen „Was gefällt Ihnen an Deutschland“ und „Was gefällt innen nicht an Deutschland“. An einer Stelle im Film kommen auch Clubmitglieder zu Wort. Vor jedem Statement wird durch die Symbole „Daumen nach oben“ 3 und „Daumen nach unten“ $\vartheta$ verdeutlich dass es sich um einen positiven bzw. negativen Aspekt handelt.

\section{Transkription des Videos Deutschland positiv negativ}

+ positiv

Junger Mann: Besonders gut? Die Autobahnen.

Susan: Warum?

Junger Mann: Warum? Weil man schnell fahren darf.

\section{- negativ}

Junge Frau mit Migrationshintergrund: Die Leute sind kalt

Björn: Ja

Junge Frau mit Migrationshintergrund: Sie wollen Keinen Kontakt haben.

+ positiv

Hakan: Äh, Deutschland ist sehr modern. Schule ist auch sehr gut. Äh, in die Schule kann nicht schlagen beim Lehrer. Und so alles, gut.

\section{- negativ}

Serife: Die Sommer gefällt mir nicht, weil es gibt net so viel Sonne. Und die und die Landratsamt gefällt mir nicht.

\section{+ positiv}

Susan: oh toll (Missfallensäußerung wegen der bisher unbefriedigenden Antworten)

Gruppe von männlichen Teenagern mit Migrationshintergrund: Ja ok die Menschen hier, die Menschen gefallen, die Freundlichkeit, Menschen, die Leben, die Freundlichkeit. Die Frauen.

\section{- negativ}

Älterer Mann: Ja manchmal die Kriminalität 


\section{+ positiv}

Susan: Wenn's Schnee gibt gefällt mir ... (unverständlich) und dass wir können Busfahren wann wir wollen.

\section{- negativ}

Meral: (türkisch)

Serife übersetzt: Die Kultur gefällt nicht.

\section{+ positiv}

Frau mit Migrationshintergrund (mit Kopftuch): Deutschland was gefällt mir, mhm das System, Disziplin... Susan: $\mathrm{mhm}$

Frau mit Migrationshintergrund (mit Kopftuch): ...die Ordnung

Hakan: Was gefällt in ihnen in Deutschland?

Mann mit Berliner Akzent: In Deutschland?

Hakan: Ja

Mann mit Berliner Akzent: Ja alles mein Gott das Leben ist so schön oder, solange kein Krieg ist gell und wir alle zu Essen und zu trinken haben $n$ Dach überm Kopf und und sie gehen ja noch zur Schule gell. Hakan: Ja

Mann mit Berliner Akzent: Sie können zur Schule gehen nicht überall kann man zur Schule gehen gell, das is ne tolle Sache. Da hab ich viel gelernt.

\section{- negativ}

Frau: Der Euro (lachen) Der Euro.

Susan: War's besser mit äh D-Mark?

Frau: D-Mark war viel besser.

Susan: OK

Schülerin (möglicherweise mit Migrationshintergrund): Die Lehrer sind ganz schön streng.

Mädchen mit Migrationshintergrund: Ich weiß net also ich kann des jetzt nur mit der Türkei vergleichen, weil in der Türkei kann man net so frei alleine als Mädchen rumlaufen und hier hat man seine Ruhe vor den Leuten. Da stresst dich halt net so viele.

Am Beispiel dieser Produktion lässt sich zeigen, wie durch aktive Medienproduktion Reflexion und Kommunikation ausgelöst werden kann.

Bei der Sichtung der Aufnahmen zu diesem Film wurde die Aussage eines Mädchens mit Migrationshintergrund, die Lehrer seien streng in Deutschland, zum Ausgangspunkt einer engagiert geführten Gruppendiskussion über Freiheit, Disziplinierungsmöglichkeiten und körperliche Bestrafung. Ein Knetanimationsfilm über Schüler, die einen Lehrer schlagen, ist im Zusammenhang mit dieser Diskussion zu sehen („School“).

Als am Ende der Praxisphase die Bewertungen der verschiedenen Dimensionen im CHICAM-Club mit einem speziellen Bilderfragebogen erhoben wurden, ergab sich folgendes Bild:

\begin{tabular}{|l|l|l|l|l|l|}
\hline & $\begin{array}{l}\text { sehr gut ge- } \\
\text { fallen }\end{array}$ & gut gefallen & mittel gefallen & $\begin{array}{l}\text { weniger gut } \\
\text { gefallen }\end{array}$ & $\begin{array}{l}\text { gar nicht ge- } \\
\text { fallen }\end{array}$ \\
\hline $\begin{array}{l}\text { Leute auf der } \\
\text { Straße inter- } \\
\text { viewen und fil- } \\
\text { men }\end{array}$ & 4 & 1 & 1 & 1 & 1 \\
\hline
\end{tabular}


Auffällig ist eine positive Bewertung von fünf Clubmitgliedern. Mittlere und negative Einschätzungen nahmen Clubmitglieder vor, die zur Zeit der Praxisphase in bezug auf Sprache und im Auftreten relativ unsicher waren.

Kurz nach dem Ausbruch des zweiten Golfkrieges im März 2003 hatten die Kinder/Jugendlichen im Club selbst das Bedürfnis geäußert, zu diesem Thema eine ähnliche Befragung zu machen. Es ging es um Stimmen und Meinungen zum Golfkrieg.

Sowohl Susan (13 Jahre, aus den USA) als auch Taskania (14 Jahre, aus der Dominikanischen Republik) machten die Erfahrung, dass sie gegenüber den ersten Kamerabefragungen auf der Straße beim zweiten Mal weniger ängstlich und mit mehr Mut und Selbstsicherheit auf fremde Menschen zugehen konnten:

Susan: Und ich finde auch dass ähm finde das auch jetzt gut aber früher nich mehr also wann ma so rumgelaufen sind im Stadt und so gefragt und so find ich gut jetzt

Peter Holzwarth: Und früher nich so?

Susan: Früher nich so da war ich da hat ich noch mehr Angst

Peter Holzwarth: Früher heißt bei dem bei dem ersten Film, wo ihr zum ersten Mal Leute angesprochen habt. mhm

Susan: Aber jetzt nich mehr.

Peter Holzwarth: mhm

Susan: Also auf letzten hab ich jede gefragt, Taskania hat auch ge...

Peter Holzwarth: Und wie kam des, dass des sich so verändert hat? Also erst so Ängstlich und jetzt ganz mutig?

Susan: Vielleicht weil des des zweite mal war. Oder dritte mal so.

Ähnlich äußert sich auch Taskania:

Peter Holzwarth: Und hast du n Unterschied gemerkt beim zweiten mal, wo's um den Irak-Krieg ging und ähm zum ersten mal wo's um Deutschland ging, was den Leuten gefällt und nicht gefällt. Also gab's $n$ Unterschied zwischen dem ersten mal wo's um Deutschland ging und dem zweiten mal wo's um den Irak-Krieg ging?

Taskania: (Lachen)

Björn Maurer: Wo du das erste mal auf Leute zugegangen bist mit der Kamera.

Taskania: Ja habe ich, habe ich ein bisschen Angst gehabt, aber zweites mal nicht mehr.

Björn Maurer: Und als wir dann noch mal losgezogen sind oder als ihr noch mal losgezogen seid mit der Kamera zum Irak? Des war ja dann...

Taskania: Ja, keine Ahnung mehr haben wir gehabt. Des war so. Hat bei uns Spaß gemacht.

Björn Maurer: Aha, Warum habt ihr so viele Interviews gemacht?

Raysa: Ja weil wir keine Ahnung gehabt haben.

Björn Maurer: Weil ihr was gehabt habt?

Taskania: Keine Ahnung ... also wir waren so ... wir waren nicht traurig und ... Die erste Mal haben wir Ahnung gehabt, aber... Ahnung, Also so

Peter Holzwarth: Ahnung?

Taskania: Miedo ${ }^{7}$, weißt du

Peter Holzwarth: Angst?

\footnotetext{
${ }^{7}$ miedo (span.): Angst
} 
Taskania: Angst, ja.

Peter Holzwarth: Und das zweite mal?

Taskania: keine mehr.

Björn Maurer: Und wenn ihr jetzt jetzt hier die Kamera nehmen müsstet und Leute befragen müsstet, wär kein Problem?

Taskania: Wär kein Problem.

Björn Maurer: Cool!

Auch in einem Interview für einen Radiobeitrag über CHICAM im Rahmen der Sendung „SWR-International“ äußerte Susan gegenüber einem Redakteur mit griechischem Mgrationshintergrund ihre Fortschritte:

Interviewer SWR-International: Wie gefällt dir denn das Projekt hier?

Susan: Isch ganz gut isch ganz toll.

Interviewer SWR-International: Mhm und was macht ihr, was gefällt dir denn so?

Susan: Mein mit Aufnehmen mit die mit die Kamera und do und auf den Computer zu gehen und Filme zu schneiden und so.

Interviewer SWR-International: Und auch auf mit den auf die Leute zugehen und dann mit den Leuten zu sprechen?

Susan: Ja jetzt isch ja no, jetzt isch schon besser, weil letztes mal hab hat hatte ich Angscht gehabt aber jetzt nemme.

\section{Literatur}

Aksoy, A. and Robins, K., 2000: Thinking across spaces: transnational television from Turkey. European Journal of Cultural Studies 3(3), pp. 343-365.

Buckingham, D., 2002: Children and Media Overview. In: In: Global Kids, Global Media: a review of research relating to children, media and migration in Europe. http://www.chicam.net/media/pdf/DEL1_part2.pdf (Zugriff: 05.03.2004), p. 99-115.

Buckingham, D. \& Harvery; I., 2003: Imagining the Audience - Reflections on the pilot phase of VideoCulture. In: Niesyto, H. (Hg.): VideoCulture. Video und interkulturelle Kommunikation. Grundlagen, Methoden und Ergebnisse eines internationalen Forschungsprojekts: München: KoPäd, S. 111-138.

Charlton, M. / Neumann, K., 1992: Medienkindheit, Medienjugend. München: Quintessenz.

Cunningham, S., 2001: Popular media as public "sphericules" for diasporic communities. International Journal of Cultural Studies 4(2): pp. 131-147.

Featherstone, M., 1995: Undoing Culture. Globalization, Postmodernism and Identity. London: Sage.

Güntürk,R., 1999: Mediennutzung der Migranten - mediale Isolation? In: Butterwegge, Ch./ Hentges, G./ Sarigöz, F. (Hg.): Medien und multikulturelle Gesellschaft. Opladen: Leske und Budrich, S. 136-143.

Hannerz, U., 1996: Transnational Connections London: Routledge.

Holzwarth, P., 2003: Symbolkompetenz im Kontext interkultureller Kommunikation mit Video. In: Medienwissenschaft Schweiz/Science de Mass Médias Suisse 2/2003, S. 75-81.

Holzwarth, P. \& Maurer, B., 2003: Kreative Bedeutungskonstruktion und ästhetische Reflexivität im Spannungsfeld von Symbolproduktion und Symbolverstehen. Eine fallbezogenen Analyse. In: Niesyto, H. (Hg.): VideoCulture. Video und interkulturelle Kommunikation. München: KoPäd, S. 139-168. 
Holzwarth, P. \& Maurer, B., 2004: Workpackage 6. Unveröffentlichtes Projektdokument. Ludwigsburg.

Holzwarth, P. \& Maurer, B., 2002: Children and Media: Germany. In: Global Kids, Global Media: a review of research relating to children, media and migration in Europe. http://www.chicam.net/research.html (Zugriff 27.7.2002), p. 117-131.

Keupp, H. u.a., 1999: Identitätskonstruktionen. Das Patchwork der Identitäten in der Spätmoderne. Reinbek bei Hamburg, Rowohlt.

Livingstone, S. and Bovill, M. (eds.), 200: Children and their Changing Media Environment. Mahwah, NJ: Lawrence Erlbaum.

Maurer, B., 2003: Migration und interkulturelle Medienarbeit. Beitrag zur medienpädagogischen Konzeption des EU Forschungsprojekts CHICAM. Unveröffentlichte Diplomarbeit. Pädagogische Hochschule Ludwigsburg.

Niesyto, H., 2003: VideoCulture - Projektentwicklung und Projektergebnisse. In: Niesyto, H. (Hg.): VideoCulture. Video und interkulturelle Kommunikation. Grundlagen, Methoden und Ergebnisse eines internationalen Forschungsprojekts: München 2003: KoPäd, S. 15-110.

Niesyto, H. (Hg.), 2001a: Selbstausdruck mit Medien. Eigenproduktionen mit Medien als Gegenstand der Kindheits- und Jugendforschung. München: KoPäd.

Niesyto, H., 2001b: Qualitative Jugendforschung und symbolischer Selbstausdruck. In: Belgrad, J. / Niesyto, H. (Hg.): Symbol. Verstehen und Produktion in pädagogischen Kontexten. Hohengehren: Schneider, S. 55-73.

Otto, H.U. / Kutscher, N. / Clepien, G., 2003: Die digitale Bildungskluft als Herausforderung für die Pädagogik. In: http://wwwhomes.uni-bielefeld.de/nkutscher/forum\% 20international\%202003\%20seiten262-283.pdf (Zugriff: 05.03.2004).

Schiffauer, W./Baumann, G./ Kastoryano, R./ Vertovec, S. (Hg.), 2002: Staat - Schule Ethnizität. Politisch Sozialisation von Immigratenkindern in vier europäischen Ländern. Münster: Waxmann.

Schorb, B., 2003: Was guckst Du, was denkst Du? - Der Einfluss des Fernsehens auf das Ausländerbild von Kindern und Jugendlichen. Kiel: Unabhängige Landesanstalt für Rundfunk und neue Medien (ULR).

Weiß, H. \& Trebbe, J., 2001: Mediennutzung und Integration der türkischen Bevölkerung in Deutschland. Ergebnisse einer Umfrage des Presse- und Informationsamtes der Bundesregierung. Potsdam.

Winter, C. / Thomas, T. / Hepp, A. (Hg.), 2003: Medienidentitäten. Identität im Kontext von Globalisierung und Medienkultur. Köln: Herbert von Halem Verlag.

\section{Anhang}

Die folgende Tabelle enthält eine Übersicht zu den Filmen, die in Deutschland entstandenen sind:

\begin{tabular}{|l|l|l|l|l|l|l|}
\hline $\begin{array}{l}\text { Name der } \\
\text { Produktion }\end{array}$ & Thema & $\begin{array}{l}\text { Darstel- } \\
\text { lung sform }\end{array}$ & Sprache & $\begin{array}{l}\text { Ge- } \\
\text { schlecht, } \\
\text { Alter und } \\
\text { Herkunft } \\
\text { der Produ- } \\
\text { zierenden }\end{array}$ & $\begin{array}{l}\text { Produktions- } \\
\text { kontext }\end{array}$ & $\begin{array}{l}\text { Besonder- } \\
\text { heiten }\end{array}$ \\
\hline $\begin{array}{l}\text { Vorstellungs- } \\
\text { video }\end{array}$ & $\begin{array}{l}\text { Der } \\
\text { CHICAM- } \\
\text { Club } \\
\text { Deutschland } \\
\text { stellt sich } \\
\text { vor }\end{array}$ & & $\begin{array}{l}\text { Deutsch, } \\
\text { Türkisch, } \\
\text { Tunesisch, } \\
\text { Englisch, } \\
\text { Spanisch }\end{array}$ & $\begin{array}{l}\text { 3 Jungen } \\
\text { und 4 Mäd- } \\
\text { chen 12-15 } \\
\text { Jahre } \\
\text { Türkei, Tu- } \\
\text { nesien, Ku- }\end{array}$ & & \\
\hline
\end{tabular}




\begin{tabular}{|c|c|c|c|c|c|}
\hline & & & & $\begin{array}{l}\text { ba, Domini- } \\
\text { kanische } \\
\text { Republik, } \\
\text { USA }\end{array}$ & \\
\hline $\begin{array}{l}\text { Deutschland } \\
\text { positiv nega- } \\
\text { tiv }\end{array}$ & $\begin{array}{l}\text { Statements } \\
\text { zu positiven } \\
\text { und negati- } \\
\text { ven Aspek- } \\
\text { ten in } \\
\text { Deutschland }\end{array}$ & Reportage & $\begin{array}{l}\text { Deutsch, 1x } \\
\text { kurz tür- } \\
\text { kisch }\end{array}$ & & \\
\hline Irak-Film & $\begin{array}{l}\text { Meinungen } \\
\text { zum } \\
\text { Irak-Krieg } \\
2003\end{array}$ & Reportage & Deutsch & & $\begin{array}{l}\text { Mit Fremd- } \\
\text { material aus } \\
\text { Nachrichten }\end{array}$ \\
\hline At the beach & $\begin{array}{l}\text { Kampf und } \\
\text { Freund- } \\
\text { schaft mit } \\
\text { einem Mon- } \\
\text { ster am } \\
\text { Strand } \\
\end{array}$ & $\begin{array}{l}\text { Knet- } \\
\text { animation }\end{array}$ & Nonverbal & $\begin{array}{l}\text { 1 Junge } \\
14 \text { Jahre } \\
\text { Tunesien }\end{array}$ & $\begin{array}{l}\text { Mit Fotos } \\
\text { vom Strand } \\
\text { aus Tunesi- } \\
\text { en (Einweg- } \\
\text { kamera) }\end{array}$ \\
\hline Bank & Banküberfall & $\begin{array}{l}\text { Knet- } \\
\text { animation }\end{array}$ & Nonverbal & & \\
\hline Dog & $\begin{array}{l}\text { Freund- } \\
\text { schaft und } \\
\text { Liebe }\end{array}$ & $\begin{array}{l}\text { Knet- } \\
\text { animation }\end{array}$ & Nonverbal & $\begin{array}{l}2 \text { Mädchen } \\
13 \text { und } 14 \\
\text { Jahre } \\
\text { USA und } \\
\text { Dominikani- } \\
\text { sche Repu- } \\
\text { blik }\end{array}$ & \\
\hline School & $\begin{array}{l}\text { Lehrer } \\
\text { schlägt und } \\
\text { Schüler } \\
\text { schlagen } \\
\text { zurück }\end{array}$ & $\begin{array}{l}\text { Knet- } \\
\text { animation }\end{array}$ & Nonverbal & $\begin{array}{l}1 \text { Mädchen } \\
\text { und } 1 \text { Junge } \\
14 \text { und } 12 \\
\text { Jahre } \\
\text { Dominikani- } \\
\text { sche Repu- } \\
\text { blik und Ku- } \\
\text { ba }\end{array}$ & \\
\hline $\begin{array}{l}\text { Freundschaft } \\
\text { und Fußball }\end{array}$ & $\begin{array}{l}\text { Freund- } \\
\text { schaft und } \\
\text { Hilfe / } \\
\text { Freund- } \\
\text { schaft und } \\
\text { Raub }\end{array}$ & $\begin{array}{l}\text { Narrativer } \\
\text { Spielfilm }\end{array}$ & Nonverbal & $\begin{array}{l}2 \text { Jungen } \\
13 \text { und } 14 \\
\text { Jahre } \\
\text { Türkei und } \\
\text { Tunesien }\end{array}$ & \\
\hline $\begin{array}{l}\text { Videoclip } \\
\text { über Schule }\end{array}$ & & Videoclip & & & \\
\hline
\end{tabular}

